\title{
Heartbeat Detection Using Oscillatory Envelope Pattern in Noisy Electrocardiogram
}

\author{
Hsiao-Lung Chan ${ }^{1,4}$, Fu-Tai Wang ${ }^{2}$, Yi-Sheng Lee ${ }^{1}$, Chun-Li Wang ${ }^{3}$ \\ ${ }^{1}$ Department of Electrical Engineering, Chang-Gung University, Taoyuan, Taiwan \\ 2 Department of Electrical Engineering, Hwa Hsia University of Technology, New Taipei, Taiwan \\ ${ }^{3}$ Department of Cardiology, Chang Gung Memorial Hospital, Taoyuan, Taiwan \\ ${ }^{4}$ Neuroscience Research Center, Chang Gung Memorial Hospital, Taoyuan, Taiwan
}

\begin{abstract}
Heartbeat detection from electrocardiogram (ECG) is crucial for heart rate, heart rate variability, and arrhythmia analysis. However, the detection usually suffers from the interference from violent body movement or muscular activity in wearable ECG devices. Here, we propose a novel heartbeat detection based on the envelope pattern of the oscillatory components derived from ECG by a bandpass filter from 18 to $20 \mathrm{~Hz}$. The envelope of the positive peaks of the emphasized signal is therefore constructed. Each adjacent QRS pair corresponds to a U-type pattern in the envelope. Then, the QRS beat is identified by the amplitude and interval of the U-wave. Meanwhile, low signal-to-noise segment is also marked by the envelope morphology for avoiding wrong recognition of an artifact as a heartbeat.
\end{abstract}

\section{Introduction}

Electrocardiogram (ECG) is a low-cost, noninvasive measure of cardiac electrical activity. ECG analysis has become a standard diagnostic tool for detecting cardiac arrhythmia. In particular, QRS detection is the first step for cardiac arrhythmia analysis. The energy-thresholding method proposed by Pan and Tompkins provides a simple and useful means of finding the occurrence of normal beats and ventricular etopic beats [1]. Template match, a similarity measure of QRS template and running-window ECG, is used to detect normal beats $[2,3]$. Incorporating with template match in the energythresholding method is able to detect QRS and discriminate ventricular etopic beats from normal beats [4]. In 2002, Lee et al demonstrated that the outer trajectory of ECG phase space is mainly contributed by QRS complex, so the area covered by phase space portrait can be used to detect the occurrence of QRS [5].

As the need of wearable ECG application in mobile health care creates an challenge that the QRS detection usually suffers from the interference from violent body movement or muscular activity. The motion artifact may have similar waveshape as QRS complexity so that it is also emphasized by bandpass filtering and differentiation in the Pan-Tompkins method, thereby generating a significant energy marker and being caught by the adaptive threshold. Although template match can find the QRS which has similar waveform as the template, the artifact may have QRS-like shape, whereby the coexistence of several high cross-correlation peaks confounds QRS identification.

In this paper, we propose a novel heartbeat detection based on the envelope pattern of the oscillatory components derived from ECG by a bandpass filter from 18 to $22 \mathrm{~Hz}$. Each adjacent QRS pair corresponds to a Utype pattern in the envelope. Therefore, each QRS beat is identified by the amplitude and interval of the U-wave to for avoiding wrong recognition of an artifact as a heartbeat.

\section{Methods}

\subsection{QRS emphasizing}

Most commonly appeared artifacts in ECG contain very-low frequency baseline wandering and highfrequency powerline interference. The afore-mentioned artifacts can be easily eliminated by a bandpass filter because their spectral content is separately distributed from that of QRS complex. Nevertheless, the artifacts due to the violent body motion and muscular contamination not only have comparable amplitude but also overlapped spectral band. Therefore, a narrower bandpass filter is needed to emphasize QRS complex.

A Butterworth lowpass filter with a cutoff frequency of $22 \mathrm{~Hz}$ and a Elliptic highpass filter with a cutoff frequency of $18 \mathrm{~Hz}$ are used to construct the QRS emphasizing filter. As shown in Fig.1, an ECG signal is first normalized between 0 and 1 (upper row):

$$
x_{\text {norm }}(n)=\left(x(n)-x_{\min }\right) /\left(x_{\max }-x_{\min }\right)
$$

The normalized ECG is subsequently filtered by the 
bandpass filter. The background activity as well as QRS complexity is chacterized by a oscillatory pattern after bandpass filtering.

The positive peaks in the oscillatory ECG are detected, whereby an ECG envelope is constructed based on the positive peaks.
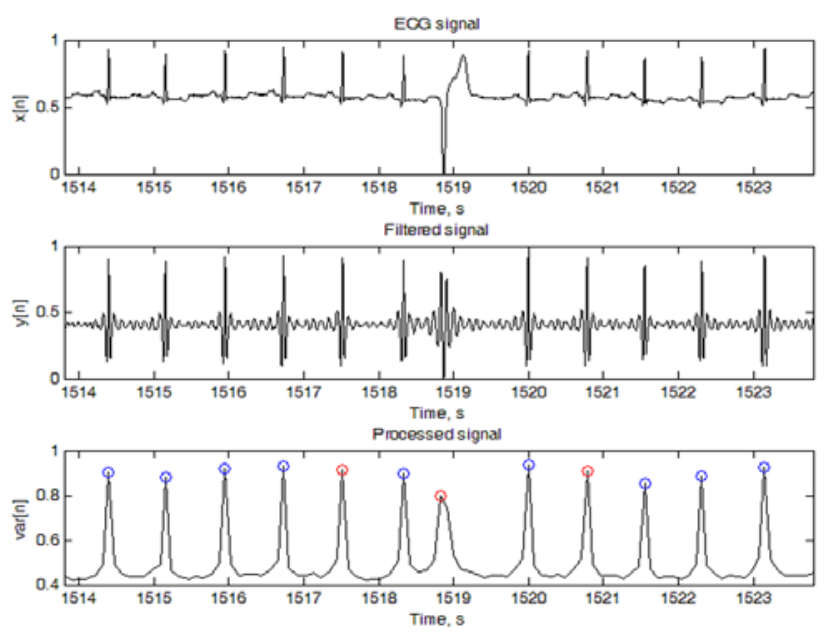

Figure 1. A ECG signal (upper) is characterized by an oscillatory pattern (middle) after bandpass filtering. An ECG envelope is therefore constructed based on the positive peaks of the oscillatory component (lower).

\subsection{QRS identification}

As depicted in Fig.1, each adjacent QRS pair corresponds to a U-type pattern in the ECG envelope. Therefore, a QRS is identified if its correspnding amplitude and interval meet one of the following criteria:

(1) The amplitude of non-QRS is less than the $15 \%$ of QRSs' amplitude;

(2) The deflection angle of U-type pattern is larger than an angle threshold.

The first criterion is used to screen the QRS beat with a high signal-to-noise ratio (SNR); the second criterion, a smooth U pattern. Figure 2 demonstrates an example of a high-qulaity ECG which has a high SNR and a smooth U-type pattern.
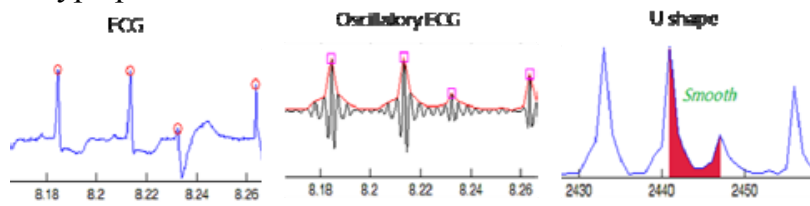

Figure 2. A high-quality ECG signal with a high sinal-tonoise ratio (left), its oscillatory component (middle), and a smooth U-type pattern (right).
If these two criteria are not met, this ECG epoch may have noisy contamination. As shown in Fig. 3, the ECG signal is contaminated by high-freuqency noises (lower). Three U-type envelopes (marked by red filling region in upper panel) are not actually formed by adjacent QRS beats but by a QRS and an artifact. Compared to the normal U-types, the deflection angle of these three Utype morphology is smaller, so they are regarded as noisy contaminated U-types. The artifact-induced peak is neglected, and the envelope is reformed by connecting to next following peak. If the new U-type satisfy the criterion of larger deflection angle, the newly included QRS beat is a heartbeat. Otherwise, the above process repeats.
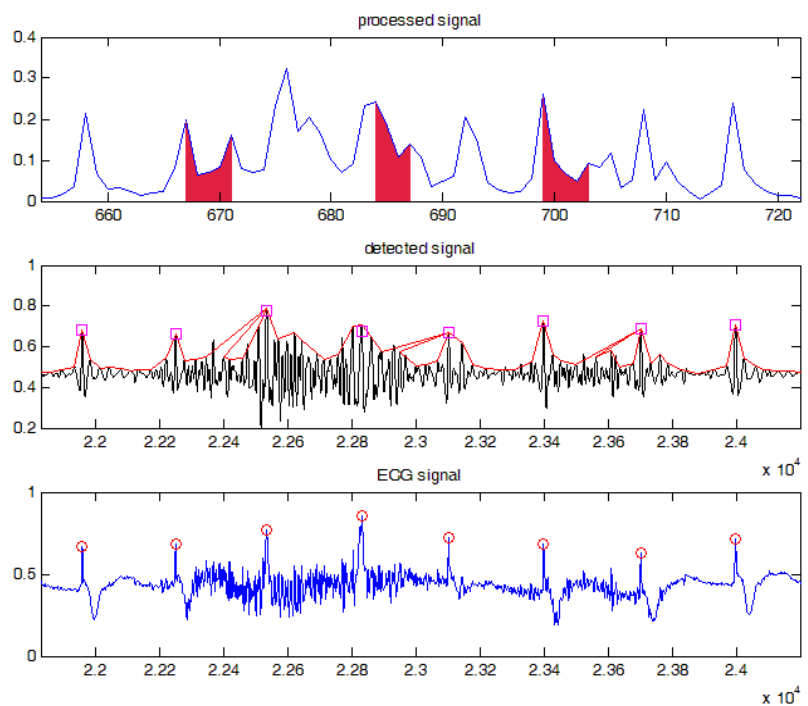

Figure 3. A ECG signal with a noise contamination (lower) produces ambiguous oscillatory compoents (mddle) whcih create three U-type envelopes whose deflection angle is less than a angle thershold (red filling region in upper panel).

\subsection{Performance evaluation}

The MIT/BIH arrhythmia database was used to assess the performance of the proposed QRS detection. The $\mathrm{MIT} / \mathrm{BIH}$ has forty-eight, 30-min, 2-lead ECG records with a sampling rate of $360 \mathrm{~Hz}$. Two records with apparent artifact contamination and one record with ventricular etopic beats were used for the illustration of the operation of the proposed method.

The accuracy is represented by sensitivity, positive prediction value (PPV), and detection error rate (DER). The sensitivity is defined as the true positive (TP) divided by total beat number $(\mathrm{N})$; the PPV, the TP divided by the sum of TP and false positive (FP); the EDR, the sum of $\mathrm{FP}$ and false negative (FN) divided by $\mathrm{N}$. 


\section{Results}

Figure 4 depicts a 10-s ECG segment with apparent muscular artifacts in Record 104. The muscular activity results in several envelop peaks whereby produces several unsmoothed U-type envelopes. Through the deflection angle identification, these unsmoothed U-types were detected and reformed by searching next envelope peaks. The QRS beats were successfully detected. The sensitivity, PPV, and DER of this record are 0.9978, 0.9951 , and 0.0072 , respectively.
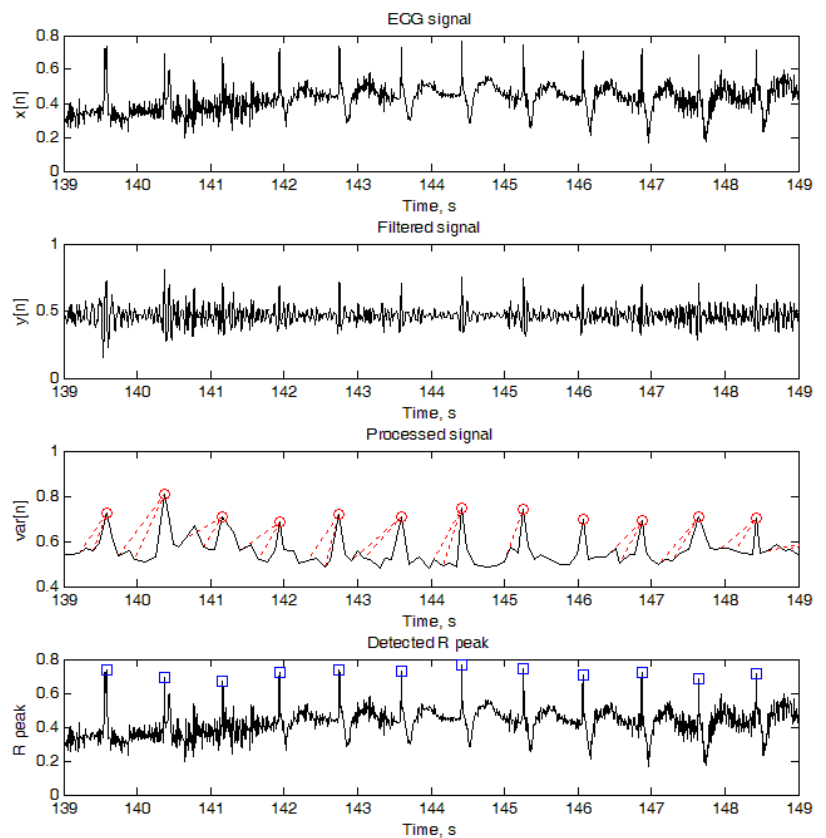

Figure 4. A 10-s ECG segment in Record 104. The muscular artifacts cause several false envelope peaks and unsmoothed U-type enevlopes (third row). After U-type reformation, the QRS beats are successfully detected.

Figure 5 depicts a 10-s ECG segment with apparent motion artifacts in Record 105. Similarly, the motion artifacts yield undesired envelop peaks. The un-smoothed U-type criterion further found these pseudo patterns and led to correct QRS detection. The sensitivity, PPV, and DER of this record are 0.9965, 0.9965, and 0.0070, respectively.

Figure 6 depicts a 10-s ECG segment with ventricular etopic beats (VEBs) in Record 106. Most VEBs as well as normal QRS are characterized by a smooth U-type envelope. However, one VEB has decreased oscillatory amplitude after QRS emphasizing because its spectral content falls beyond the passband of the emphasizing filter. Therefore, this VEB was miss-detected (indicated by FN). The sensitivity, PPV, and DER of this record are $0.9857,0.9876$, and 0.0266 , respectively.
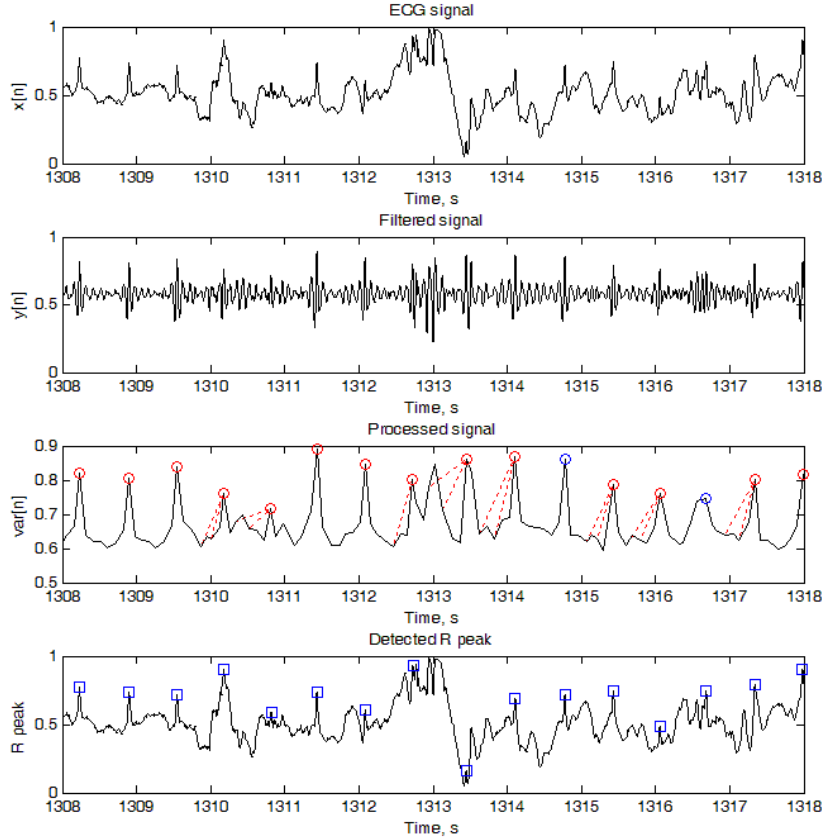

Figure 5. A 10-s ECG segment in Record 105. The motion artifacts yield undesired envelop peaks and unsmoothed U-types. After U-type reformation, QRS beats are correctly marked.
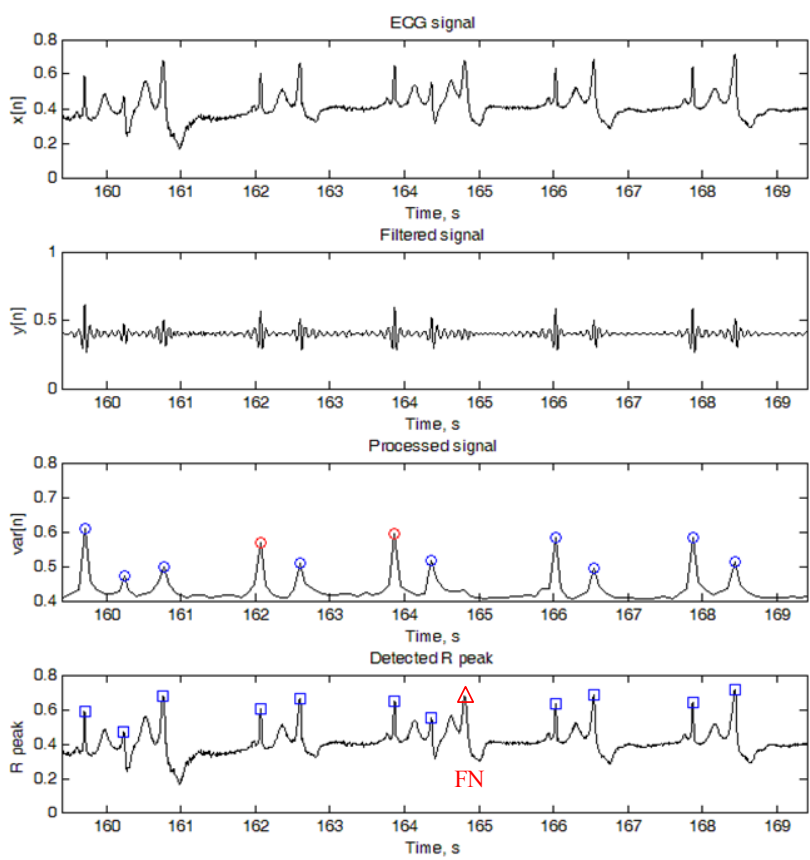

Figure 6. A 10-s ECG segment in Record 106. Most ventricular etopic beats as well as normal QRS have a smooth U-type envelope but an etopic beat is misdetected (indicated by FN) because of its decreased oscillatory amplitude after QRS emphasizing. 


\section{Discussion}

The adopted bandpass filter has a narrow bandwidth which characterizes the ECG signal by an oscillatory component whereby QRS complexes are emphasized and baseline wandering, $\mathrm{P}$ and $\mathrm{T}$ waves are eliminated. Although muscular interferences and motion artifacts also appears as an oscillatory pattern, further identification of U-type envelope and U-type reformation can avoid incorrect identification as a QRS beat. Nevertheless, some VEBs whose frequency content fall beyond the passband of the emphasizing filter. Readjustment of the specification of the emphasizing filter or the adoption of multi-band QRS emphasizing is needed in future work.

\section{Acknowledgements}

The authors would like to give sincere appreciation to the grant supports from the Chang Gung Memorial Hospital, Taiwan under Contract CMRPD2E0051 and the Minister of Science and Technology, Taiwan under Contract MOST 105-2221-E-182-035.

\section{References}

[1] Pan J, Tompkins WJ. A real-time QRS detection algorithm. IEEE Trans Biomed Eng 1985; 32:230-6.

[2] Dobbs SE, Schmitt NM, Ozemek HS. QRS detection by template matching using real-time correlation on a microcomputer. J Clin Eng 1984; 9:197-212.

[3] Rosado A, Bataller M, Guerrero JF, Calpe J, Francés JV, Magdalena JR. High performance hardware correlation coefficient assessment using programmable logic for ECG signals. Microprocess Microsy 2003: 27:33-9.

[4] Chan HL, Chou WS, Chen SW, Fang SC, Liou CS, Hwang YS. Continuous and on-line analysis of heart rate variability. J Med Eng Technol 2005; 29:227-34.

[5] Lee JW, Kim KS, Lee B, Lee B, Lee MH. A real time QRS detection using delay-coordinate mapping for the microcontroller implementation. Ann Biomed Eng 2002; $30: 1140-51$.

Address for correspondence.

Hsiao-Lung Chan, $\mathrm{PhD}$.

Dept Electrical Engineering, Chang-Gung University, 259 Wen-Hwa 1st Road, Kwei-Shan, Tao-Yuan 333, Taiwan.

chanhl@mail.cgu.edu.tw. 